\title{
Novel Gravity Distortion Evaluation on Large Antenna Array for Deep Space Communication
}

\author{
Miguel A. Salas Natera, Ramón Martínez Rodríguez-Osorio, Leandro de Haro Ariet, and Miguel \\ Calvo Ramón.
}

\begin{abstract}
This paper presents a simple gravity evaluation model for large reflector antennas and the experimental example for a case study of one uplink array of $4 \times 35-m$ antennas at $X$ and Ka band. This model can be used to evaluate the gain reduction as a function of the maximum gravity distortion, and also to specify this at system designer level. The case study consists of one array of 35-m antennas for deep space missions. Main issues due to the gravity effect have been explored with Monte Carlo based simulation analysis.
\end{abstract}

Keywords - Gravity effect, antenna arrays, large antenna, and error analysis.

\section{INTRODUCTION}

$\mathrm{R}$ EGARDING the increasing number of satellite and constelations launches, the improvement of the data download capacity is required. As well as in Earth orbits, the number of satellites has been increased in Deep Space Mission, where the Deep Space Network (DSN) from the Jet Propulsion Laboratory of the National Aeronautics and Space Administration (JPL-NASA) and Deep Space Antennas (DSA) from European Space Tracking (ESTRACK) network will be saturated. Thus, new space communication technologies such as Ground Station Systems have been under development during the last decade.

This case study has been defined in line with the project in [1] where the feasibility of new uplink arrays has been analyzed and, calibration challenges covered. Furthermore, the analysis has been done to one uplink array of $4 \times 35-\mathrm{m}$ antennas at $\mathrm{X}$ and $\mathrm{Ka}$ band. System requirements were based on previous work by NASA for the Deep Space Network Array [2].

The propagation of $35-\mathrm{m}$ antennas errors over the gain of the antenna array in uplink has been studied. The evaluation of losses of the array gain response based on Monte Carlo simulation $[3,4]$ with information from works presented in $[5,6,7]$ is presented. This paper is focused on the model for antenna efficiency reduction as a function of the gravity effect. The gravity model is based on the expansion of the model presented in [11] and [12] for a first mode distortion of the antennas surface to obtain the antenna efficiency reduction. In addition to the evaluation of a 35-m antenna array, this model has been contrasted with the gravity distortion model proposed by JPL $[8,9]$.

Results of the errors analysis are useful for system designers to evaluate the performance of the antenna array, give information about the required system, and specify antenna array components or sub-systems tolerances presented in an error budget. The error analysis starts with the antenna gain model represented with the Ishikawa diagram in Fig. 1. Tolerances of the system can be better defined by analyzing the impact of each antenna errors on the gain loss of the antenna array.

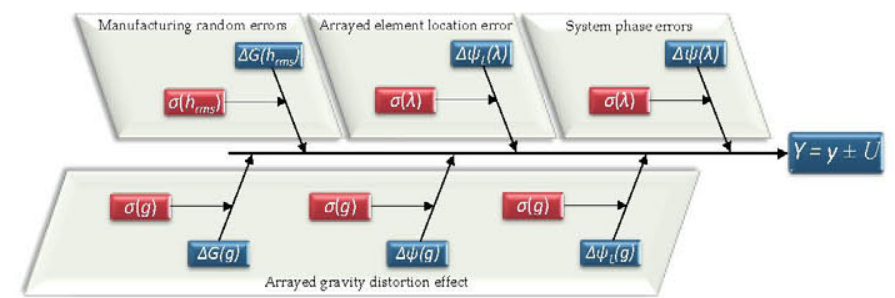

Fig. 1. Ishikawa diagram to express the relationship between error sources and antenna array

The error analysis can be divided in two parts. Part 1 about each antenna and, part 2 about the $4 \times 35-\mathrm{m}$ antenna array. There is a list of system parameters which have impact on the error budget such as the variable gain control performance, the antenna distortion due to the manufacturing random errors, the gravity effect, phase and gain errors of the system, the phase error of the arrayed signals, the phase error due to temperature variation, etc. Classifying surface errors fall into two main categories [10]: time-invariant panel mechanical manufacturing errors $\left(h_{r m s}\right)$ and panel-setting errors at the rigging angle and time-varying errors induced by gravity, wind, and thermal effects.

Regarding the antenna formulation and the goal of an error budget analysis, main errors and distortion sources are listed in Table I. Note that, one antenna element only includes the manufacturing random error contribution and the gravity distortion effect.

TABLE I

ERRORS AND DISTORTION SOURCES IN ANTENNA AND ANTENNA ARRAY One Antenna Element Array of $4 \times 35 \mathrm{~m}$ Antennas

1. Manufacturing random errors $\Delta G\left\{\sigma\left(h_{r m s}\right)\right\}$.

2. Gravity distortion effect.

a. Phase center variation. i. Gain variation $\Delta G$

ii. Phase variation $\Delta \psi$.

\begin{tabular}{l} 
Array of $4 \times 35 m$ Antennas \\
\hline 1. Manufacturing random errors $\Delta G\left\{\sigma\left(h_{r m s}\right)\right\}$. \\
2. Gravity distortion effect. \\
a. Phase center variation. \\
i. Gain variation $\Delta G\{\sigma(g)\}$. \\
ii. Phase variation $\Delta \psi\{\sigma(g)\}$. \\
b. Phase center error $\Delta \psi_{L}\{\sigma(g)\}$. \\
3. Arrayed signals. \\
a. Arrayed element location error \\
b. $\quad \Delta \psi_{L}\{\sigma(\lambda)\}$. \\
bystem phase error $\Delta \psi\{\sigma(\lambda)\}$.
\end{tabular}


The gravity model has been fitted for larger antenna dishes of $35-\mathrm{m}$ diameter with a third order polynomial regression also applied for 6 and $12 \mathrm{~m}$ diameter antennas. Furthermore, the results from the gravity distortion evaluation have been contrasted with the JPL model in $[8,9]$ obtaining good results.

This paper is organized as follows: Section II explains the model for evaluation of the gravity effect. Section III presents the results of the evaluation of the gravity effect on the case study. Finally, in section IV, conclusions of this work are listed.

\section{GAIN VARIATION MODEL FOR GRAVITY EFFECT}

The gravity model is based on the distortion model presented in [11,12] and presented in equation (1) for one antenna.

$$
\Delta \mathrm{z}=\delta_{\max }\left(\frac{2 \rho}{\mathrm{D}}\right)^{3} \cos (\mathrm{Mg} \varphi)
$$

Where $\delta_{\max }$ is the peak error on the main reflector surface due to the gravity distortion. $\mathrm{D}$ is the diameter of the main reflector. $M g$ is the modal index related with the spatial variation of the distortion. $\rho$ is the radial distance to the symmetry axis (z). Finally, $\varphi$ is the angle between the vector of the radial distance and the $\mathrm{X}$ axis.

The gravity model with the first mode $(M g=1)$ of distortion [13] has been fitted for larger antenna dishes of 35$\mathrm{m}$ diameter with a third order polynomial equation. The equation (1) was solved for 6 and 12 meters antennas and GRASP (TICRA) simulations have been performed to obtain the efficiency reduction model then fitted for 35-m antennas.

The maximum gravity error $\varepsilon_{\max }$ for simulations in this work is $1.1 \mathrm{~mm}$. Thus, the gravity distortion model for DSA $35-\mathrm{m}$ antennas can be expressed in terms of the reduction of the antenna efficiency as presented in equations (2) and (3) for $\mathrm{Ka}$ and $\mathrm{X}$ band, respectively.

$$
\begin{aligned}
\Delta \eta_{\mathrm{a}} \mathrm{Ka}\left(\varepsilon_{\max }\right)= & -0.12578 \cdot \varepsilon_{\max }{ }^{3}- \\
& 0.08969 \cdot \varepsilon_{\max }{ }^{2}+0.006455 \cdot \varepsilon_{\max }+1 \\
\Delta \eta_{\mathrm{a}} \mathrm{X}\left(\varepsilon_{\max }\right)= & 5.027 \times 10^{-4} \cdot \varepsilon_{\max }{ }^{3}- \\
& 0.00103 \cdot \varepsilon_{\max }{ }^{2}+0.000711 \cdot \varepsilon_{\max }+1
\end{aligned}
$$

Following, equation (4) from [8] is used to generate gain versus elevation curves. The gain values are referenced to the feed horn aperture so different configurations will have the same gain values.

$\mathrm{G}(\theta)=\mathrm{G}_{0}-\mathrm{G}_{1}(\theta-\gamma)^{2}-\frac{\mathrm{A}_{\text {zen }}}{\sin (\theta)}[\mathrm{dBi}]$

Where $\gamma$ is the angle at which the antenna has been previously compensated for gravity distortion $(\gamma=45$ in this case study). For validation of the gravity model, $G_{1}$ in equation (4) can be estimated as the ratio between the undistorted and the distorted antenna gain at $G_{a n t}\left(90^{\circ}\right)$ as follows
$\mathrm{G}_{1}=\left(\frac{\mathrm{G}_{0}-\mathrm{G}_{\text {ant }}\left(90^{\circ}\right)}{(90-\gamma)^{2}}\right)$

$G_{a n t}\left(90^{\circ}\right)$ in term of the antenna efficiency reduction at $\varepsilon_{\max }$ can be expressed as follows

$\mathrm{G}_{\text {ant }}\left(90^{\circ}\right)=10 \cdot \log 10\left(\eta_{a} \cdot \Delta \eta_{a}\left(\varepsilon_{\max }\right)\left(\frac{\pi \mathrm{D}}{\lambda}\right)^{2}\right)$

$G_{o}$ is the antenna peak gain calculated using the Ruze formula from [14].

$\mathrm{G}_{0}=10 \cdot \log 10\left(\eta_{\mathrm{a}}\left(\frac{\pi \mathrm{D}}{\lambda}\right)^{2}\right)-4.343 \cdot\left(\frac{4 \pi \mathrm{h}_{\mathrm{rms}}}{\lambda}\right)^{2}$

\section{RESULTS OF THE GRAVITY EFFECT EVALUATION}

\section{A. Validation of the proposed model for gravity effect}

Results from the gravity distortion model have been contrasted with the model proposed by JPL [8,9] in Fig. 3 .

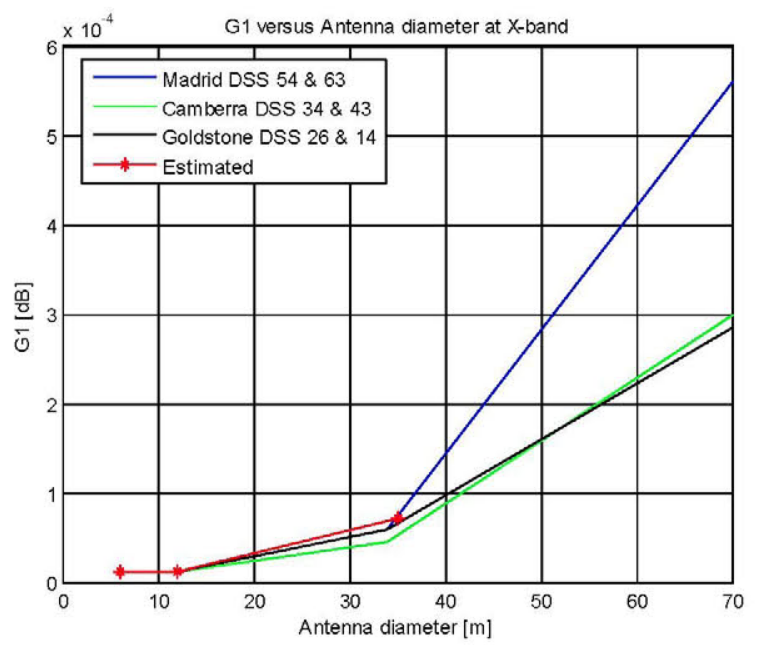

(a) X band

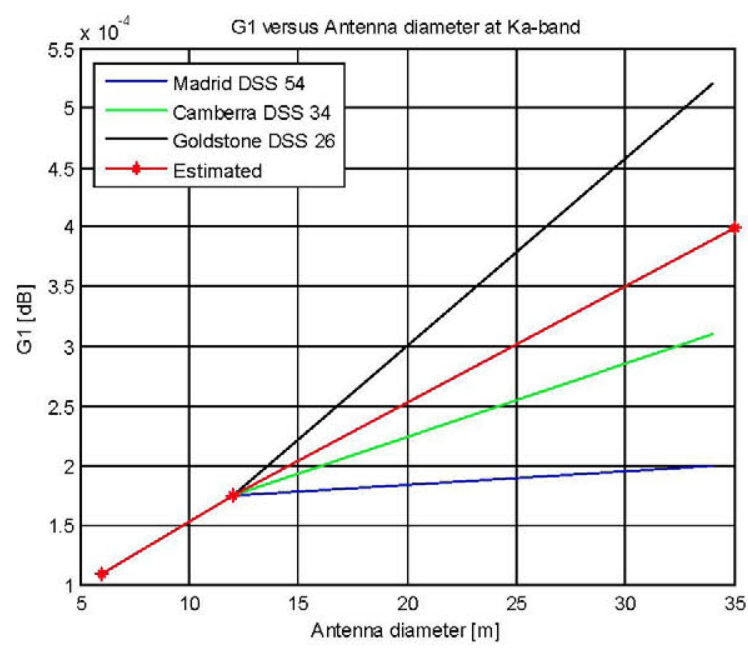

(b) Ka band

Fig. 3. Gl versus antenna diameter $\left(\varepsilon_{\max }=1.1 \mathrm{~mm}\right)$ 
Fig. 3 shows the validation of the third order equations proposed to estimate the reduction of the antenna efficiency contrasting G1 values of 34 and $70 \mathrm{~m}$ antennas of Madrid, Goldstone, and Camberra, to those G1 values of the analyzed antennas of 6,12 [3], and the 35-m antennas of this case study.

The G1 value estimated for the $35-\mathrm{m}$ antenna fulfills the expected G1 value of the proposed model by JPL as depicted in Fig. 3. Data for the $70 \mathrm{~m}$ diameter antenna has been found only for $\mathrm{X}$ band, while the 34-m diameter antenna has available data for $\mathrm{X} / \mathrm{Ka}$ bands operating mode. Furthermore, results depicted in Fig. 3 shows good results also for the estimated G1 values of 6 and 12 meters antennas.

The gain $G_{o}$ values of the undistorted $35-\mathrm{m}$ antenna with $h_{r m s}=0 \mathrm{~mm}$ are $68.66 \mathrm{dBi}\left(\eta_{a}=67.2 \%\right)$ and $79.83 \mathrm{dBi}\left(\eta_{a}=\right.$ $61 \%$ ) for $\mathrm{X}$ and Ka band, respectively.

\section{B. Gain variation}

Before arraying, Fig. 4 shows results for both mean values of maximum gravity error and its standard deviation related to the gain loss of one large reflector antenna using equations (2), (3), and (4).

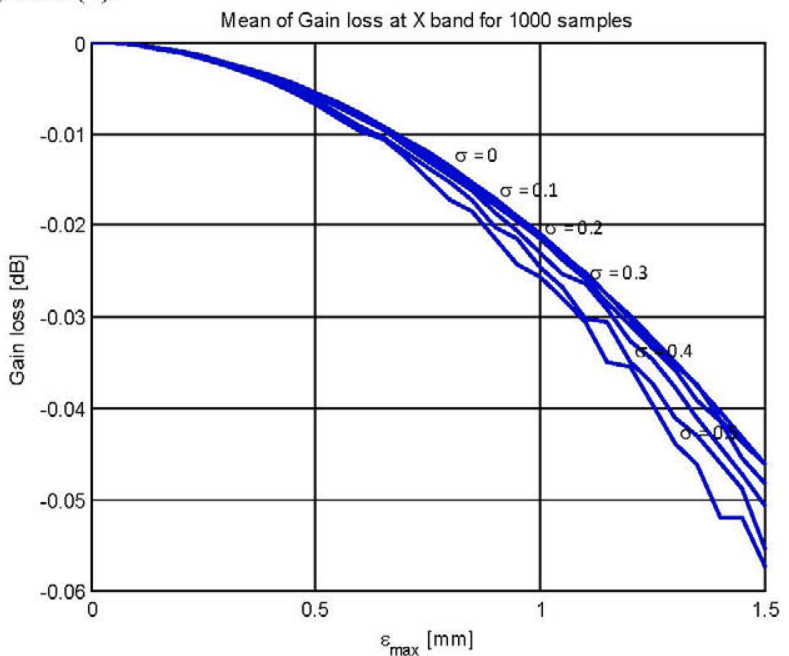

(a) $\mathrm{X}$ band

Mean of Gain loss at Ka band for 1000 samples

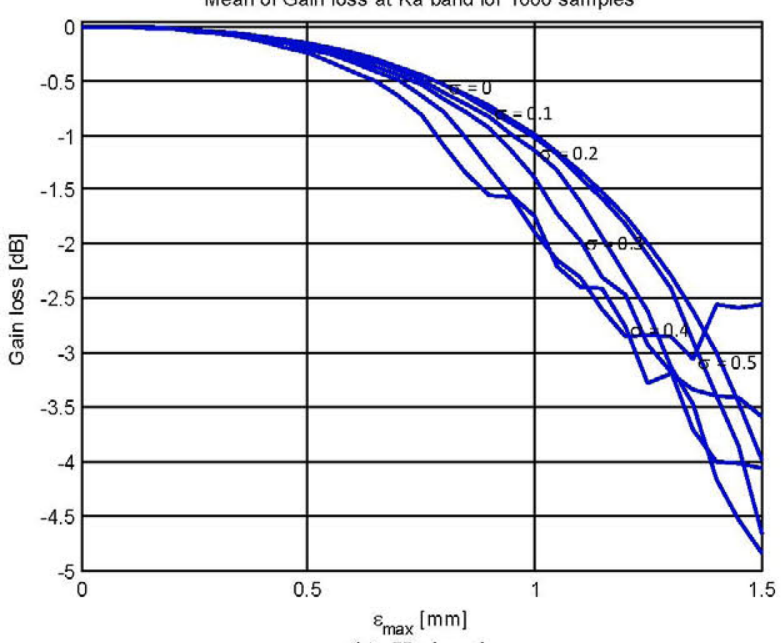

(b) Ka band

Fig. 4. Gain loss due to gravity effect as a function of $\varepsilon_{\max }$ and $\sigma$

For Monte Carlo simulation, the gain loss due to gravity effect is estimated using one random vector with Gaussian distribution, standard deviation, and the mean value relative to the gravity error at the maximum gain loss (equation 6).

Fig. 4.b shows results for Ka band. In this case, the model of equation (3) has an undesirable behavioral farther than an standard deviations equal to $0.4 \mathrm{~mm}(\sigma \geq 0.4 \mathrm{~mm})$ and a maximum gravity distortion of $1.3 \mathrm{~mm}\left(\varepsilon_{\max } \geq 1.3 \mathrm{~mm}\right)$ due to the third order of the fitted model. Thus, the range of standard deviation and maximum gravity distortion error is reasonable to be equal lower than 0.4 and $1.3 \mathrm{~mm}$, respectively.

Fig. 5 shows the estimated mean value of the gain loss of the antenna array for $\mathrm{X}$ and $\mathrm{Ka}$ band simulations as a function of the maximum gravity error and $h_{r m s}=0.34 \mathrm{~mm}$.

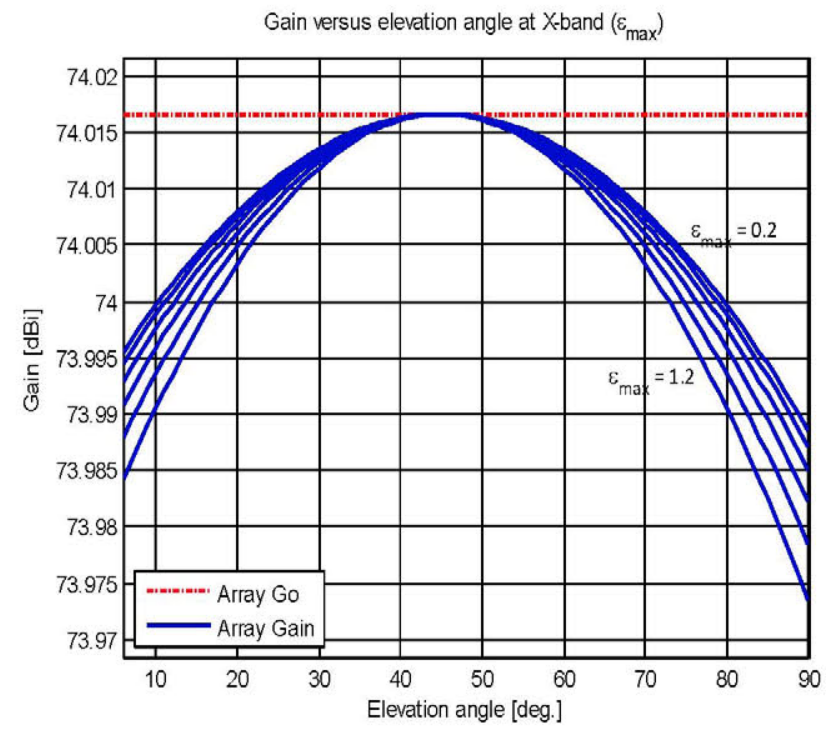

(a) X band

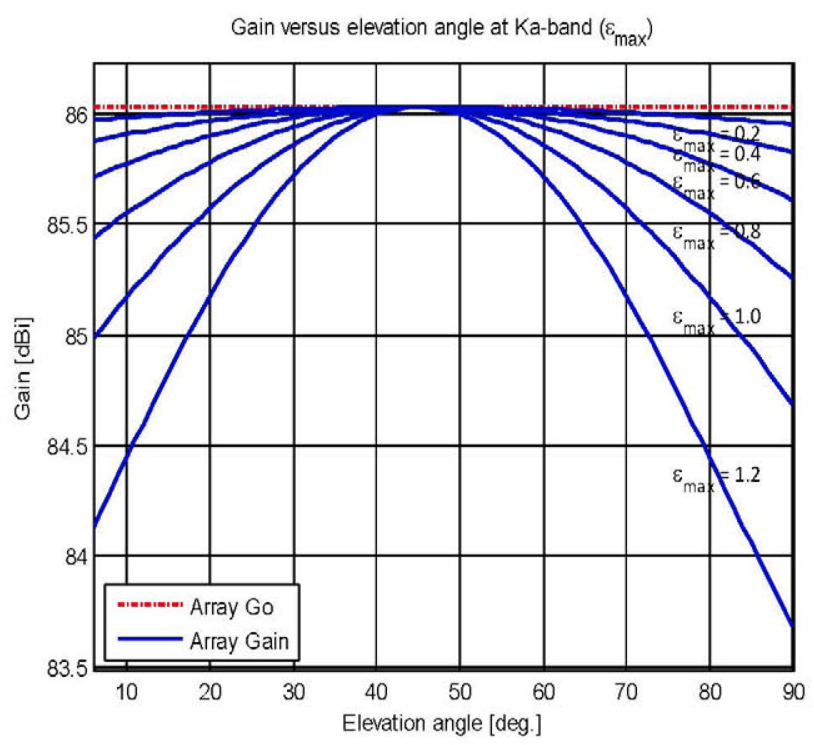

(b) Ka band

Fig. 5. Gain variation due to gravity effect as a function of $\varepsilon_{\max }$ and $\sigma$

The study of the errors propagation is an important task of 
the analysis with the antenna array model $Y$ (see Fig. 1). In many cases, $Y$ is not measured but an estimation of the measurand or output quantity $y$ can be estimated for design purposes. This estimation can be done from $L$ other quantities $x_{1}, x_{2}, x_{3}, \ldots, x_{L}$ with a given relation $y=f\left(x_{1}, x_{2}, x_{3}, \ldots, x_{L}\right)$ [15].

\section{CONCLUSION}

Extensive Monte Carlo simulations have been done to analyze the propagation of the gravity effect in the $35-\mathrm{m}$ Antenna Array. The model for evaluation of the antenna efficiency reduction due to gravity effect on 34-m antennas proposed has been contrasted with the gravity distortion model proposed by the Jet Propulsion Laboratory (JPL).

Results of errors analysis simulations are useful for system designers to evaluate the performance of the antenna array, and gives information about the required system tolerances and specification of the antenna array components or subsystems.

\section{ACKNOWLEDGMENT}

Authors wish to thank CICYT (Comisión Interministerial de Ciencia y Tecnología) under projects SICOMORO (ref:TEC-2011-28789-C02-01), and INSA (Ingeniería y Servicios Aeroespaciales S.A.) for the partial funding of this work.

\section{REFERENCES}

[1] INSA, "Proposal to ESA for Future Architecture of ESA Deep Space Stations for Enhanced Mission Support," INSA FAST-INSA-OFE-0025-09 v1.0, March 2009.

[2] S. D. Slobin and T. T. Phan, "34-m HEF Subnet Telecommunications Interfaces," California Institute of Technology DSN Telecommunications Link Design Handbook 103, Rev. B, Sept. 2008.

[3] M. Calvo Ramón and L. De Haro Ariet, "Future Architecture of ESA Deep Space Station for Enhance Mission Support," Universidad Politécnica de Madrid Study of 6 and $12 \mathrm{~m}$ antennas FAST UPM D1 Part 1 WP3120, Febrary 2011.

[4] M. Calvo Ramón and M. A. Salas Natera, "Future Architecture of ESA Deep Space Station for Enhance Mission Support," Universidad Politécnica de Madrid Study of calibration techniques and error budget analysis for an antenna array of four $35 \mathrm{~m}$ antennas FAST UPM D2 v1.3, March 2011.

[5] Y. Rahmat-Samii, R. A. Hoferer, and H. Mosallaei, "Beam Efficiency of Reflector Antennas: The Simple Formula," IEEE Antennas and Propagation Magazine, vol. 40, no. 5, pp. 82-87, Oct. 1998.

[6] L. R. D'Addario, "Combining Loss of a Transmitting Array due to Phase Errors," Jet Propulsion Laboratory, California Institute of Technology, Pasadena, California, Report IPN Progress Report 42-175, Nov. 2008.

[7] F. Amoozegar, L. Paal, A. Mileant, and D. Lee, "Analysis of Errors for Uplink Array of 34-m Antennas For Deep Space Applications," in Proc. IEEE Aerospace Conference, Big Sky, MT, March 2005, pp. 1235-1257.

[8] S. D. Slobin, "70-m Subnet Telecommunications Interfaces," California Institute of Technology DSN Telecommunications Link Design Handbook 101, Rev. C, Sept. 2009.

[9] S. D. Slobin and T. Pham, "34-m BWG Stations Telecommunications Interfaces," California Institute of Technology DSN Telecommunication Link Design Handbook 104, Rev. F, June 2010.

[10] W. A. Imbriale, Large Antennas of the Deep Space Network, J. H. Yuen, Ed. Pasadena, California, EEUU: Deep-Space Communications and Navigation Series, 2002.

[11] Y. Rahmat-Samii, "Array Feeds for Reflector Surface Distortion Compensation: Concepts and Implementation," IEEE Antennas and Propagation Magazine, vol. 32, no. 4, pp. 20-26, Aug. 1990.

[12] R. A. Hoferer and Y. Rahmat-Samii, "Subreflector Shaping for Antenna Distortion Compensation: An Efficient Fourier-Jacobi Expansion with GO/PO Analysis," IEEE Transactions on Antennas and Propagation, vol. 50, no. 12, pp. 1676-1686, Dec. 2002.

[13] L. De Haro Ariet and M. Calvo Ramón, "Future Architecture of ESA Deep Space Station for Enhance Mission Support," Universidad Politécnica de Madrid, FAST Deliverable, FAST UPM D1 Part 2 - WP3120, Febrary 2011.

[14] ITU-R, "Reference antenna patterns of large-aperture space research service earth station to be used for compatibility analysis involving a large number os distributed interference entries in the bands $31.8-32.3$ $\mathrm{GHz}$ and 37.0 - $38.0 \mathrm{GHz}, "$ The International Telecommunication Union Recommendation Rec. ITU-R SA.1811, 2007.

[15] B. N. Taylor and C. E. Kuyatt, "Guidelines for evaluating and expressing the uncertainty of NIST measurement results," National Institute of Standards and Technology NIST Technical Note 1297, 1994.

[16] Y. T. Lo, "On the Beam Deviation Factor of a Parabolic Reflector," IEEE Transactions on Antennas and Propagation, vol. 8, no. 3, pp. 347-349, May 1960.

[17] J. Baars, "Characteristics of a reflector antenna," ESO NRAO Electronic, Tucson, Internal Report ALMA Memo 456, 2003.

[18] C. A. Balanis, Antenna Theory: Analysis and Design, 3rd ed. New York: John Wiley \& Sons, 2005.

[19] F. Davarian, "Uplink Arrays for the Deep Space Network," Proceedings of the IEEE, vol. 95, no. 10, pp. 1923-1930, Oct. 2007. 